CORRIGENDUM

doi:10.1038/nature07881

\title{
A burst of segmental duplications in the genome of the African great ape ancestor
}

Tomas Marques-Bonet, Jeffrey M. Kidd, Mario Ventura,

Tina A. Graves, Ze Cheng, LaDeana W. Hillier, Zhaoshi Jiang, Carl Baker, Ray Malfavon-Borja, Lucinda A. Fulton,

Lynne V. Nazareth ${ }^{1}$, Donna M. Muzny ${ }^{1}$, Can Alkan, Gozde Aksay,

Santhosh Girirajan, Priscillia Siswara, Lin Chen,

Maria Francesca Cardone, Arcadi Navarro, Richard A. Gibbs ${ }^{1}$,

Elaine R. Mardis, Richard K. Wilson \& Evan E. Eichler

${ }^{1}$ Human Genome Sequencing Center, Baylor College of Medicine, One Baylor Plaza, Houston, Texas 77030, USA.

Nature 457, 877-881 (2009)

In this Letter, Lynne V. Nazareth, Donna M. Muzny and Richard A. Gibbs were inadvertently omitted from the author list. 